\title{
Evaluation of an Automatic Insulated Isothermal PCR System for Rapid and Reliable Field-Deployable Detection of African Swine Fever Virus
}

\section{TRAN THI HUYEN NGA}

VNU University of Science, Vietnam National University - Hanoi

\section{LE THI NHI-CONG}

Institute of Biotechnology, Vietnam Academy of Science and Technology

\section{PHAM BANG PHUONG}

Thai Nguyen University of Agriculture And Forestry

\section{LUU VAN QUYNH}

Kim Nguu instrument and chemical import/export JSC.

\section{Viet-Linh Nguyen ( $\nabla$ nvlinh@ibt.ac.vn )}

Institute of Biotechnology, Vietnam Academy of Science and Technology https://orcid.org/0000-00027472-1543

\section{Research Article}

Keywords: African Swine Fever, ASFV detection, diagnosis, iiPCR, POCKIT Central, real-time PCR

Posted Date: June 2nd, 2021

DOl: https://doi.org/10.21203/rs.3.rs-531911/v1

License: (1) This work is licensed under a Creative Commons Attribution 4.0 International License. Read Full License 


\section{Abstract}

In the present study, we evaluate an automatic sample-to-answer insulated isothermal PCR system for rapid and reliable field-deployable detection of African swine fever virus in comparison to that of OIE recommended real-time PCR counterparts with samples collected in Vietnam. For analytical sensitivity, the system could detect ASFV up to a dilution of $10^{6}$ whereas the real time PCR systems could detect up to a dilution of $10^{5}$ to $10^{6}$. For specificity test, the system showed high specificity to ASFV in compare to different other types of swine pathogens: PRRSV, FMDV, PCV2, CSFV. The diagnostic performance comparison on 6 different types of samples showed $97.3 \%$ to $100 \%$ agreement with reference real-time PCR. The results of this study indicated that POCKIT Central-based insulated isothermal PCR system is a rapid, reliable and sample-flexible method for effective detection of ASFV.

\section{Introduction}

African swine fever (ASF), caused by African swine fever virus (ASFV), was a severe pig disease originated from Africa, initially mostly being severe in several sub-Saharan countries and being endemic in some European regions until 2007, 2014 and 2018-2019 European and Asian outbreaks. As being an extremely contagious and fatal viral disease, in just less than one month since the first outbreak in August 2018, ASF had been spreading in 25 provinces of China and nationwide expanding to 10 other Asian countries with millions of pigs being culled despite the efforts of governments in ASF control (Food and Agriculture Organization of the United Nations, 2019). Because there is no effective treatment or vaccine, ASF control will only be successful with exact determination of disease outbreaks, herd culling and serious sanitary practice in non-infected regions (Cisek et al., 2016; Rock, 2017). Therefore, a rapid and reliable test is a crucial tool in a national strategy to prevent and control the epidemic.

There are two main approaches for ASFV diagnostic tests. The first one includes virus isolation, hemadsorption test, viral genome detection, and real-time PCR, which are all accurate, determinative but also usually expensive, instrument- and good technique-required. The other includes ELISA and IFA (Cubillos et al., 2013; OIE, 2012; Simulundu et al., 2018), and isothermal amplification techniques such as RPA, LAMP and CPA (Oura et al., 2013) which are cheaper and more rapid, easier to deploy even on-field, but most is limited in sensitivity and accuracy. It is still in concern whether there is a method which is rapid, field-deployable with moderate instrument, and appropriate accuracy for effective detection of ASFV.

Insulated isothermal PCR (iiPCR) is a modified PCR technique that based on Rayleigh-Bénard convection. The principle of the method is still the PCR one with steps of denaturation, annealing, and extension carried out in a capillary vessel (R-tube; GeneReach) which is heated through the bottom end and fluid containing target DNA/cDNA and all amplification materials are cycling through different temperature levels inside the tube to complete steps of PCR continuously and simultaneously (Chang et al., 2012, Tsai et al., 2012). With POCKIT ${ }^{\mathrm{TM}}$ Nucleic Acid Analyzer (GeneReach), a field-deployable device, the iiPCR process is carried out with automatic DNA/RNA amplification and detection, making the detection of 
pathogen easy, convenient and rapid. Moreover, using the POCKIT ${ }^{\mathrm{TM}}$ Central PCR System with magnetic bead-based DNA/RNA extraction integrated, the time consumed from sample to result for detection of pathogens is shortened to 1-1.5 hours with simplified interpretation of iiPCR results in the form of "positive" or "negative" readouts (Chang et al., 2012). To date, iiPCR assays with POCKITT instruments or system have been used for the detection of many human and animal pathogens (Tsai et al., 2018; Lauterbach et al., 2018), etc. In the present study, we evaluate the ability of the integrated POCKIT ASFV Reagent Set using POCKIT ${ }^{\mathrm{TM}}$ Central PCR System to detect ASFV in comparison to the reference real-time PCR on samples collected in pigs with and without ASF in Northern and Southern Vietnam.

\section{Materials And Methods Sample collection}

Samples for this study were provided independently from two institutions of RAHO-6 (Southern Vietnam) and NCVD (Northern Vietnam) to carry out experiments.

For analytical sensitivity test, two ASFV positive-predetermined samples identified by reference real-time PCR with Ct of 19.7 (tissue homogenate) and 14.0 (blood) were provided by RAHO-6 and NCVD, respectively. Dilutions were made by adding sufficient volume of fresh phosphate buffer saline (PBS) with samples for POCKIT Central System before extraction, and of deionized water with DNA samples after extraction for real-time PCR systems.

For analytical specificity test, 2 samples, in the form of cell culture medium, which are ASFV negative and either CSFV-, PRRSV-, and FMDV-positive were provided by RAHO-6 for each case, and 2 samples, in the form of cell culture medium for CSFV and tissue homogenized solution with the rest, which are ASFV negative and either CSFV-, PRRSV- and PCV2-positive were provided by NCVD for each case, respectively (total 6 samples per institution).

For diagnostic performance comparative study, ASFV positive-, ASFV negative-predetermined samples and untested samples from different tissues were provided by NCVD and RAHO-6. All samples, except whole blood samples, were frozen after collection and stored at $-20^{\circ} \mathrm{C}$ until use.

Prior to extraction of nucleic acid, $0.1 \mathrm{~g}$ tissue of each sample was well grinded in $1 \mathrm{ml}$ PBS, then

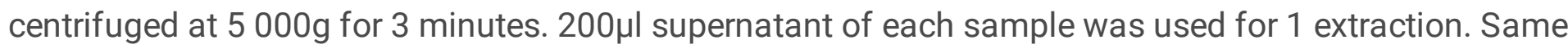
volume $(200 \mu \mathrm{l})$ of each whole blood and serum sample went directly to nucleic acid extraction without any pretreatment.

\section{Nucleic acid extraction for real-time PCR}

At RAHO-6, nucleic acid extraction was done with Thermo Scientific KingFisher Purification system (Thermo Scientific, USA) using chemicals and reagents purchased from Thermo Scientific following manufacturer's guidelines, resulting $200 \mu \mathrm{l}$ of extracted nucleic acid solution. At NCVD, nucleic acid extraction was done on a Taco Nucleic Acid Automatic Extraction System (GeneReach Biotech, Taiwan) 
with Taco DNA/RNA Extraction kit (GeneReach Biotech, Taiwan) according to the manufacturer's instructions. Briefly, $200 \mu \mathrm{l}$ of the sample was pipetted into the first well of the extraction plate prior to the process of automatic extraction resulted $200 \mu \mathrm{l}$ of nucleic acid solution. All nucleic acids were collected individually and maintained at $-80^{\circ} \mathrm{C}$ until further use.

For analytical sensitivity tests, 2 and 3 extractions were performed per sample for RAHO-6 and NCVD, respectively. For analytical specificity and diagnostic performance comparative study, there was only 1 extraction per sample.

\section{Real-time PCR}

At RAHO-6, real-time PCR were carried out with Platinum ${ }^{\text {TM }}$ Quantitative PCR SuperMixUDG (Thermo Scientific, USA), primers and probe for ASFV detection primers according to standard recommendation of OIE (King et al., 2003; OIE, 2012) by QuantStudio 5 (Thermo Scientific, USA) real-time thermocycler. Amplification conditions used were an initial denaturation step of $94^{\circ} \mathrm{C}$ for $120 \mathrm{~s}$ followed by 45 cycles of $94^{\circ} \mathrm{C}$ for $30 \mathrm{~s}, 58^{\circ} \mathrm{C}$ for $60 \mathrm{~s}$ and $72^{\circ} \mathrm{C}$ for $45 \mathrm{~s}$, and an elongation at $72^{\circ} \mathrm{C}$ for $420 \mathrm{~s}$ before an indefinite holding at $4^{\circ} \mathrm{C}$. At NCVD, real-time PCR were carried out with a commercialized real-time PCR master mix: qScript XLT 1-Step RT-qPCR ToughMix (Quantabio, USA) by ABI 7500 (Applied Biosystem, USA), primers and probe, amplification conditions were similar to those at RAHO-6. Signals are analyzed by build-in softwares of QuantStudio 5 and ABI 7500, respectively. Sample is considered positive when measured Ct is less than 40 .

\section{ASFV detection by POCKIT Central System}

ASFV detection was carried out by POCKIT Central, which is a sample-in-answer-out nucleic acid testing system by integrating magnetic bead-based nucleic acid purification, robotic arm liquid handling, and insulated isothermal PCR (iiPCR) technologies to offer a fully automatic molecular detection platform. For nucleic acid extraction and purification, POCKIT Central Cartridge Set (B) (24 tests/set) was used, including preloaded magnetic bead-based nucleic acid extraction reagents (Extraction Cartridge (B)) and Consumables for nucleic acid extraction, liquid transfer, and iiPCR amplification (Transfer Cartridge). For iiPCR amplification, POCKIT Central ASFV Premix Reagent (24 tests/set) containing Premix (lyophilized pellet) of dNTPs, primers, probe, and enzyme necessary for iiPCR amplification. Briefly, a $200 \mu \mathrm{l}$ of supernatant of homogenate sample was transferred into the first well of the extraction cassette containing lysis buffer and $95 \%$ ethanol. Extraction and iiPCR steps which focuses on the nucleocapsid vp72 gene with amplification size of 88 bps designed for all 24 ASFV genotypes were subsequently automatically carried out by the POCKIT Central reveal the test results in about 85 minutes. The device automatically collected fluorescent signal of each sample and control before and after iiPCR to make signal-to-noise $(\mathrm{S} / \mathrm{N})$ ratios and by a build-in algorithm converted into simplified results as positive $(+)$, negative (-) or others: (?) - inconclusive reaction, suggesting retest; (!) - warning; (*) - abnormal, signals outside detection range.

\section{Experimental design}


Analytical sensitivity test was carried out by comparing detection competence of the iiPCR and reference real-time PCR with 10 -fold serial dilutions of 10 times $\left(10^{1}\right.$ to $\left.10^{10}\right)$ of one ASFV positive sample, with 2 and 3 repeats each dilution at RAHO- 6 and NCVD, respectively. An ASFV predetermined positive sample and a negative control (deionized water) are added to each experiment carried out.

Analytical specificity test was carried out for 4 other swine virus pathogens (2 positive samples per pathogen of CSFV, FMDV, PCV2, and PRRSV) independently at RAHO-6 and NCVD (3 pathogens per institution) in comparison with real-time PCR. An ASFV predetermined positive sample and a negative control (deionized water) are added to each experiment carried out.

Diagnostic performance comparative study was carried out independently at RAHO-6 and NCVD with distinctive samples from each institution. Only 1 extraction per sample, and 1 repeat per sample was double tested by both iiPCR and real-time PCR. An ASFV predetermined positive sample and a negative control (deionized water) are added to each experiment carried out. If there is a discrepant sample i.e. the test result is not the same by iiPCR and real-time PCR, or the real-time test result of the pre-determined sample is not in accordance to the information provided by the sample provider, the sample would be tested in triplicate again by two methods as in discrepancy test.

\section{Statistical analysis}

Real-time PCR results were considered the standard for iiPCR, and the number of true/false positive and negative were based on agreement of iiPCR to real-time PCR in each case tested. Cohen's kappa values were used as the indicator for the degree of agreement between iiPCR and real-time PCR. Sensitivity is defined as number of true positives/ (number of true positives + number of false negatives). Specificity is defined as number of true negatives / (number of true negatives + number of false positives). Data on $\mathrm{S} / \mathrm{N}$ and $\mathrm{Ct}$ values are provided as mean $\pm \mathrm{SD}$.

\section{Results}

\section{Analytical sensitivity}

The endpoint dilution for detection of ASVF was $10^{6}$ with POCKIT Central iiPCR System at both institutions, whereas with real-time counterparts, it was $10^{6}$ at RAHO-6 and only $10^{5}$ at NCVD (Table 1 ). It might indicate that iiPCR performed by POCKIT Central System could be as analytically sensitive as the "gold standard" real-time PCR. 
Table 1

Analytical sensitivity of POCKIT Central iiPCR System in ASFV detection

\begin{tabular}{|c|c|c|c|c|c|c|c|c|}
\hline \multirow{4}{*}{$\begin{array}{l}\text { Titer (dilution) } \\
10^{1}\end{array}$} & \multicolumn{8}{|c|}{ Detection rate (no. of positive result/total no. of assays) } \\
\hline & \multicolumn{4}{|c|}{ RAHO-6 (mean \pm SD) } & \multicolumn{4}{|c|}{ NCVD (mean \pm SD) } \\
\hline & \multicolumn{2}{|c|}{ iiPCR (S/N) } & \multicolumn{2}{|c|}{ real-time PCR (Ct) } & \multicolumn{2}{|c|}{ iiPCR (S/N) } & \multicolumn{2}{|c|}{ real-time PCR (Ct } \\
\hline & $2 / 2$ & $\begin{array}{l}3.99 \pm \\
0.25\end{array}$ & $2 / 2$ & $\begin{array}{l}22.09 \pm \\
0.25\end{array}$ & $3 / 3$ & $\begin{array}{l}3.86 \pm \\
0.29\end{array}$ & $3 / 3$ & $\begin{array}{l}18.87 \pm \\
0.26\end{array}$ \\
\hline $10^{2}$ & $2 / 2$ & $\begin{array}{l}3.91 \pm \\
0.02\end{array}$ & $2 / 2$ & $\begin{array}{l}22.02 \pm \\
0.20\end{array}$ & $3 / 3$ & $\begin{array}{l}3.54 \pm \\
0.09\end{array}$ & $3 / 3$ & $\begin{array}{l}22.05 \pm \\
0.11\end{array}$ \\
\hline $10^{3}$ & $2 / 2$ & $\begin{array}{l}3.95 \pm \\
0.08\end{array}$ & $2 / 2$ & $\begin{array}{l}24.82 \pm \\
0.07\end{array}$ & $3 / 3$ & $\begin{array}{l}2.81 \pm \\
0.60\end{array}$ & $3 / 3$ & $\begin{array}{l}28.74 \pm \\
0.24\end{array}$ \\
\hline $10^{4}$ & $2 / 2$ & $\begin{array}{l}3.56 \pm \\
0.15\end{array}$ & $2 / 2$ & $\begin{array}{l}29.07 \pm \\
0.44\end{array}$ & $3 / 3$ & $\begin{array}{l}3.12 \pm \\
0.64\end{array}$ & $3 / 3$ & $\begin{array}{l}28.18 \pm \\
1.28\end{array}$ \\
\hline $10^{5}$ & $2 / 2$ & $\begin{array}{l}3.63 \pm \\
0.27\end{array}$ & $2 / 2$ & $\begin{array}{l}33.72 \pm \\
0.45\end{array}$ & $3 / 3$ & $\begin{array}{l}3.74 \pm \\
0.31\end{array}$ & $3 / 3$ & $\begin{array}{l}33.14 \pm \\
1.33\end{array}$ \\
\hline $10^{6}$ & $2 / 2$ & $\begin{array}{l}2.46 \pm \\
0.11\end{array}$ & $2 / 2$ & $\begin{array}{l}37.19 \pm \\
0.64\end{array}$ & $3 / 3$ & $\begin{array}{l}3.18 \pm \\
0.63\end{array}$ & $0 / 3$ & $\mathrm{~N} / \mathrm{A}$ \\
\hline $10^{7}$ & $0 / 2$ & $\begin{array}{l}1.03 \pm \\
0.01\end{array}$ & $0 / 2$ & N/A & $0 / 3$ & $\begin{array}{l}1.00 \pm \\
0.02\end{array}$ & $0 / 3$ & $\mathrm{~N} / \mathrm{A}$ \\
\hline $10^{8}$ & $0 / 2$ & $\begin{array}{l}1.03 \pm \\
0.00\end{array}$ & $0 / 2$ & N/A & $0 / 3$ & $\begin{array}{l}1.00 \pm \\
0.00\end{array}$ & $0 / 3$ & $\mathrm{~N} / \mathrm{A}$ \\
\hline $10^{9}$ & $0 / 2$ & $\begin{array}{l}1.02 \pm \\
0.01\end{array}$ & $0 / 2$ & N/A & $0 / 3$ & $\begin{array}{l}1.00 \pm \\
0.00\end{array}$ & $0 / 3$ & $\mathrm{~N} / \mathrm{A}$ \\
\hline $10^{10}$ & $0 / 2$ & $\begin{array}{l}1.03 \pm \\
0.01\end{array}$ & $0 / 2$ & N/A & $0 / 3$ & $\begin{array}{l}1.00 \pm \\
0.00\end{array}$ & $0 / 3$ & $\mathrm{~N} / \mathrm{A}$ \\
\hline Positive control & $1 / 1$ & 3.62 & $1 / 1$ & 33.04 & $1 / 1$ & 4.10 & $1 / 1$ & N/A \\
\hline $\begin{array}{l}\text { Negative } \\
\text { control }\end{array}$ & $0 / 1$ & 1.00 & $0 / 1$ & N/A & $0 / 1$ & 1.03 & $0 / 1$ & N/A \\
\hline
\end{tabular}

\section{Analytical specificity}

The POCKIT Central iiPCR System for ASVF could detect the pathogen and did not cross-react with the four other popular swine pathogens of similar symptoms (Table 2). This might be an assurance that the combined system of POCKIT Central machine and kit could work properly without any compromise with other popular viral swine diseases. 
Table 2

Analytical specificity of POCKIT Central iiPCR System in ASFV detection

\begin{tabular}{|lllll|}
\hline Pathogen & \multicolumn{3}{l}{ Detection rate (no. of positive result/total no. of assays) } \\
\cline { 2 - 5 } & RAHO-6 & NCVD \\
\cline { 2 - 5 } & iiPCR & real-time PCR & iiPCR & real-time PCR \\
\hline CSFV & $0 / 2$ & $0 / 2$ & $0 / 2$ & $0 / 2$ \\
\hline PPRSV & $0 / 2$ & $0 / 2$ & $0 / 2$ & $0 / 2$ \\
\hline FMDV & $0 / 2$ & $0 / 2$ & - & - \\
\hline PCV2 & - & - & $0 / 2$ & $0 / 2$ \\
\hline ASVF (positive control) & $1 / 1$ & $1 / 1$ & $1 / 1$ & $1 / 1$ \\
\hline NTC (negative control) & $0 / 1$ & $0 / 1$ & $0 / 1$ & $0 / 1$ \\
\hline
\end{tabular}

\section{Diagnostic performance comparative study}

To evaluate the diagnostic performance of the POCKIT Central iiPCR System in ASFV detection in comparing to real-time PCR, 336 determined and undetermined samples were tested: 216 samples at RAHO-6 and 150 samples at NCVD. Diagnostic performance results were shown in Table 3. Statistical analysis was done independently for data from RAHO-6 and NCVD, as shown in Table 4. 
Table 3

Comparative diagnostic performance at RAHO-6 and NCVD

\begin{tabular}{|c|c|c|c|c|}
\hline \multirow[t]{3}{*}{ Sample type } & \multicolumn{4}{|c|}{ Detection rate (no. of positive result/total no. of assays) } \\
\hline & \multicolumn{2}{|c|}{ RAHO-6 } & \multicolumn{2}{|l|}{ NCVD } \\
\hline & iiPCR & real-time PCR & iiPCR & real-time PCR \\
\hline ASFV positive lymph node & $31 / 31$ & $31 / 31$ & - & - \\
\hline ASFV negative lymph node & $0 / 29$ & $0 / 29$ & - & - \\
\hline ASFV positive whole blood & $30 / 30$ & $30 / 30$ & - & - \\
\hline ASFV negative whole blood & $0 / 30$ & $0 / 30$ & - & - \\
\hline ASFV positive serum & $30 / 30$ & $30 / 30$ & $31 / 31$ & $31 / 31$ \\
\hline ASFV negative serum & $0 / 30$ & $0 / 30$ & $0 / 29$ & $0 / 29$ \\
\hline ASFV positive tissue homogenate & - & - & $37 / 37$ & $37 / 37$ \\
\hline ASFV negative tissue homogenate & - & - & $1 / 23$ & $0 / 23$ \\
\hline ASFV positive spleen & $30 / 30$ & $30 / 30$ & - & - \\
\hline ASFV negative spleen & $0 / 6$ & $0 / 6$ & - & - \\
\hline Untested tonsil * & - & - & $20 / 24$ & $20 / 24$ \\
\hline Untested spleen & - & - & $6 / 6$ & $5 / 6$ \\
\hline
\end{tabular}


Table 4

Summary of diagnostic performance at RAHO-6 and NCVD

\begin{tabular}{|c|c|c|c|c|c|c|c|}
\hline \multicolumn{2}{|c|}{ Test method and result } & \multicolumn{6}{|c|}{ Real-time PCR } \\
\hline & & \multicolumn{3}{|l|}{ RAHO-6 } & \multicolumn{3}{|l|}{ NCVD } \\
\hline & & Positive & Negative & Total & Positive & Negative & Total \\
\hline \multirow{5}{*}{$\begin{array}{l}\text { POCKIT } \\
\text { Central }\end{array}$} & Positive & 121 & 0 & 121 & 91 & 3 & 94 \\
\hline & Negative & 0 & 95 & 95 & 1 & 55 & 56 \\
\hline & Total & 121 & 95 & 216 & 92 & 58 & 150 \\
\hline & & \multicolumn{3}{|l|}{ RAHO-6 } & \multicolumn{3}{|l|}{ NCVD } \\
\hline & & Value & \multicolumn{2}{|c|}{$\begin{array}{l}95 \% \text { Confidence } \\
\text { Interval }\end{array}$} & Value & \multicolumn{2}{|c|}{$\begin{array}{l}95 \% \text { Confidence } \\
\text { Interval }\end{array}$} \\
\hline \multicolumn{2}{|l|}{ Sensitivity } & $100.0 \%$ & \multicolumn{2}{|c|}{$97.2-100.0 \%$} & $98.9 \%$ & \multicolumn{2}{|c|}{$95.5-100.0 \%$} \\
\hline \multicolumn{2}{|l|}{ Specificity } & $100.0 \%$ & \multicolumn{2}{|c|}{$97.8-100.0 \%$} & $94.8 \%$ & \multicolumn{2}{|c|}{$87.9-100.0 \%$} \\
\hline \multicolumn{2}{|l|}{ Accuracy } & $100.0 \%$ & \multicolumn{2}{|c|}{$98.8-100.0 \%$} & $97.3 \%$ & \multicolumn{2}{|c|}{$94.3-100.0 \%$} \\
\hline \multicolumn{2}{|c|}{ Cohen's kappa (к) } & \multicolumn{3}{|l|}{1.00} & \multicolumn{3}{|l|}{0.94} \\
\hline
\end{tabular}

At RAHO-6, the comparative diagnostic performance by both POCKIT and real-time PCR assays showed no discrepancy, revealing a 100\% sensitivity (95\% confidence interval [CI95\%], 97.2-100.0\%), 100\% specificity (CI95\%, 97.8-100.0\%). The overall agreement between the two assays was $100 \%$ (CI95\%, 98.8-100.0\%; $K=1.0$ ). At NCVD, there were 4 cases with discrepancy ( 3 false positive, 1 false negative). The comparative diagnostic performance between the two methods showed a sensitivity of $98.9 \%$ (Cl95\%, 95.5-100.0\%), 94.8\% specificity (Cl95\%, 87.9-100.0\%), and accuracy was $97.3 \%$ (CI95\%, 94.3$100.0 \% ; \mathrm{K}=0.94)$.

\section{Discrepancy tests}

There was no discrepancy in pre-determined samples i.e. no real-time test result of the pre-determined sample was different from the information provided by the sample provider. There were 4 cases of discrepancy of iiPCR to real-time PCR, all were retested. The result is shown in Table 5. 
Table 5

Summary of discrepancy test at NCVD

\begin{tabular}{|c|c|c|c|c|c|c|}
\hline \multirow[t]{2}{*}{ Case } & \multirow[t]{2}{*}{ Discrepancy } & \multicolumn{2}{|c|}{ POCKIT Central } & \multicolumn{2}{|c|}{ Real-time PCR } & \multirow[t]{2}{*}{ Final conclusion } \\
\hline & & $S / N$ & Result & Ct & Result & \\
\hline 19S1463 & PK (+) & $1.57 \pm 0.62$ & $(+)$ & UT & $(-)$ & False positive \\
\hline (tissue) & $\mathrm{RT}(-)$ & & & & & \\
\hline D13963 & PK (+) & $1.02 \pm 0.03$ & $(-)$ & UT & $(-)$ & False negative \\
\hline (tonsil) & $\mathrm{RT}(-)$ & & & & & \\
\hline D13957 & PK (-) & $1.03 \pm 0.00$ & $(-)$ & UT & $(-)$ & True negative \\
\hline (tonsil) & $\mathrm{RT}(+)$ & & & & & \\
\hline S13271 & PK (+) & $1.88 \pm 0.56$ & $(+)$ & UT & $(-)$ & False positive \\
\hline (spleen) & $\mathrm{RT}(-)$ & & & & & \\
\hline
\end{tabular}

\section{Discussion}

Without a vaccine or treatment available, infected herd culling, isolation of infected area, strict practice of sanitary is crucial for ASF controlling (Galindo and Alonso, 2017), in which ASFV diagnosis plays a central role. The less accurate and the longer the diagnosis is, the more spreading and affecting ASF becomes. In the present study, we evaluate the possibility of using POCKIT Central System for rapid and appropriately accurate detection of ASFV. With only exception of the grinding step, samples were processed automatically in a build-in system to reveal results, which could prevent personal errors and sample contamination.

The analytical sensitivity has showed low detection limit of POCKIT Central iiPCR System, which is comparable or even little better than that of real-time PCR, the reference method. The detection ranges of iiPCR system were both $10^{1}$ to $10^{6}$ dilution, similar to that at RAHO-6 whereas, at NCVD, that of real-time PCR was $10^{1}$ to $10^{5}$ only (Table 2). Moreover, the analytical specificity tests at RAHO-6 and NCVD showed that iiPCR system has good specificity to ASFV and no cross-reaction with other swine pathogen: PRRSV, FMDV, PCV2and especially CSFV - the pathogen which could make undistinguishable symptoms to that of ASFV, similarly to the results from those of real-time PCR. The results showed that the POCKIT Central iiPCR system is an analytically sensitive and specific method for ASFV detection, making it reliable and comparable to the reference method - real-time PCR.

Diagnostic performance tests were carried out with a large amount of samples collected from epidemic regions by RAHO- 6 and NCVD, with a wide range of types of samples. All criteria of sensitivity, specificity 
and accuracy was very high at both test sites with high agreement with the reference methods ( $100 \%$ at RAHO-6; $97.3 \%$ at NCVD) with high $\mathrm{k}$ values of 1.0 and 0.94 at RAHO-6 and NCVD, respectively (Table 4). For pre-determined samples, there was only one false positive in an ASFV negative homogenate sample, in which $\mathrm{Ct}$ of real-time PCR was under threshold. The discrepancy tests were 2 positives per 3 repeats (Case 19S1463, Table 5, mean S/N value is 1.57). It is remarkable that at NCVD, the analytical sensitivity of POCKIT Central System is higher than that of real-time PCR, therefore in this case, the virus concentration of the discrepant sample might be just in the detectable limit of POCKIT Central System but out of that of real-time PCR. Similarly, with case S13271, for which the mean of S/N values is 1.88 , the pathogen is undetectable to real-time PCR. Nevertheless, as considering real-time PCR the standard in a comparative study, we still conclude true or false positive/negative of our iiPCR system based on realtime PCR results. For the untested spleen and tonsil samples, the comparative study revealed 2 false positive and 1 false negative. The discrepancy 3-repeat comparative tests done afterward confirmed that the negative case was true negative (Table 5). This might increase the sensitivity and accuracy at NCVD up to $100 \%$ and $98.0 \%$, respectively (analysis not shown).

Timeliness of a national strategy for ASF epidemic control now mostly relies on the time for detection of ASFV, primarily based on test duration. Total time for the POCKIT Central System for detection of ASFV is about 2 hour including sample pretreatment of 0.5 hour, DNA extraction and iiPCR 1.5 hour, to the final results of positive or negative. It is comparably much shorter than that of real-time PCR system, which consumes at least 4 hours, including 0.5 hour for sample pretreatment, 1 hour for DNA extraction, and at least 2.5 hours for real-time PCR. Moreover, the time consumed for having the detection results by realtime PCR must include the duration of transfer of samples from the suspected farm or husbandry household to an equipped laboratory, which usually locates in modern large cities. This may last from 1 to 20 days depending on the distance between the farm/household to the laboratory. As ASF is a very contagious disease, that period of time is enough for ASF to much wider spread.

Collected data have proved that iiPCR by POCKIT Central System could be successfully applied in a wide range of types of samples with good sensitivity, specificity and accuracy. iiPCR using POCKIT Central System might be the average and appropriate point in all the aspects of test time, sensitivity, specificity, accuracy, and field deploy-ability.

In conclusion, the automatic ASFV detection iiPCR using POCKIT Central System was evaluated. It was proven to be rapid, effective with high sensitivity, specificity and accuracy in compare with the reference real-time PCR method on various sample types collected in both Northern and Southern of Vietnam. Moreover, it is user-friendly and field deployable with automatic combined NA extraction and iiPCR diagnostic detection, as well as easy manipulation and simplified result output. The system has a great potential to be apply on field screening towards effective prevention and control of ASF in Vietnam and other countries.

\section{Declarations}




\section{Funding}

Not applicable.

\section{Conflicts of interest/Competing interests}

The authors declare no financial or commercial conflict of interest.

\section{Availability of data and material (data transparency)}

Not applicable.

\section{Code availability}

Not applicable.

\section{Authors' contributions}

- Conceptualization: Viet Linh Nguyen;

- Methodology: Huyen Nga Tran Thi, Nhi Cong Le Thi, Bang Phuong Pham, Van Quynh Luu;

- Formal analysis and investigation: Huyen Nga Tran Thi, Van Quynh Luu;

- Writing - original draft preparation: Viet Linh Nguyen, Huyen Nga Tran Thi, Nhi Cong Le Thi;

- Writing - review and editing: Viet Linh Nguyen; Funding acquisition: Viet Linh Nguyen, Nhi Cong Le Thi;

- Resources: Van Quynh Luu, Bang Phuong Pham;

- Supervision: Viet Linh Nguyen.

\section{Ethics approval}

The present study was conducted in accordance with IBT institutional principles for ethics in biological research and rules for biosafety assurance. Collection and provide of samples by RAHO-6 and NCVD were officially in compliance with the national standard for pathogenic anatomy and sampling approved by the Vietnam Ministry of Agriculture and Rural Development (TCVN 8402:2010). The manuscript does not contain clinical studies or patient data.

\section{Consent to participate (include appropriate statements)}

All authors consent to participate to the research.

\section{Consent for publication (include appropriate statements)}

All authors consent for publication of the research. 


\section{References}

Achenbach, J.E., Gallardo, C., Nieto-Pelegrín, E., Rivera-Arroyo, B., DegefaNegi, T., Arias, M., Jenberie, S., Mulisa, D.D., Gizaw, D., Gelaye, E., Chibssa, T.R., Belaye, A., Loitsch, A., Forsa, M., Yami, M., Diallo, A., Soler, A., Lamien, C.E., Sánchez-Vizcaíno, J.M., 2017. Identification of a new genotype of African swine fever virus in domestic pigs from Ethiopia. Transboundary and emerging diseases, 64(5), 1393-1404.

Chang, H.F., Tsai, Y.L., Tsai, C.F., Lin, C.K., Lee, P.Y., Teng, P.H., Su, C., Jeng, C.C., 2012. A thermally baffled device for highly stabilized convective PCR. Biotechnology journal 7, 662-666.

Cisek, A.A., Dabrowska, I., Gregorczyk, K.P., Wyzewski, Z., 2016. African swine fever virus: a new old enemy of Europe. Annals of parasitology 62, 161-167.

Cubillos, C., Gomez-Sebastian, S., Moreno N, Nuñez, M.C., Mulumba-Mfumu, L.K., Quembo, C.J., Heath, L., Etter, E.M.C., Jori, F., Escribano, J.M., Blanco, E., 2013. African swine fever virus serodiagnosis: a general review with a focus on the analyses of African serum samples. Virus Research 173, 159-167.

Food and Agriculture Organization of the United Nations, 2019. ASF situation in Asia update. http://www.fao.org/ag/againfo/programmes/en/empres/ASF/2019/Situation_update_2019_12_19.html. Accessed 5 April 2020;

Galindo, I., Alonso, C., 2017. African Swine Fever Virus: A Review. Viruses. 9, 103.

King, D.P., Reid, S.M., Hutchings, G.H., Grierson, S.S., Wilkinson, P.J., Dixon, L.K., Bastos, A.D., Drew, T.W., 2003. Development of a TaqMan PCR assay with internal amplification control for the detection of African swine fever virus. Journal of Virological Methods 107, 53-61.

Lauterbach, S.E., Nelson, S.N., Nolting, J.M., Trujillo, J.D., Richt, J.A., Bowman, A.S., 2018. Evaluation of a Field-Deployable Insulated Isothermal Polymerase Chain Reaction Nucleic Acid Analyzer for Influenza A Virus Detection at Swine Exhibitions. Vector-Borne and Zoonotic Diseases 19, 212-216.

OIE, 2012. African swine fever. Manual of Diagnostic Tests and Vaccines for Terrestrial Animals 2012, Chapter 2.8.12012.

Oura, C.A., Edwards, L., Batten, C.A., 2013. Virological diagnosis of African swine fever-comparative study of available tests. Virus Research 173, 150-158.

Rock, D.L., 2017. Challenges for African swine fever vaccine development perhaps the end of the beginning. Veterinary Microbiology 206, 52-58.

Simulundu, E., Sinkala, Y., Chambaro, H.M., Chinyemba, A., Banda, F., Mooya, L.E., Ndebe, J., Chitanga, S., Makungu, C., Munthali, G., Fandamu, P., Takada, A., Mweene, A.S., 2018. Genetic characterisation of African swine fever virus from 2017 outbreaks in Zambia: Identification of p72 genotype II variants in domestic pigs. Onderstepoort Journal of Veterinary Research 85: e1-e5. 
Tsai, J.J., Liu, L.T., Lin, P.C., Tsai, C.Y., Chou, P.H., Tsai, Y.L., Chang, H.G., Lee, P.A., 2018. Validation of the Pockit Dengue Virus Reagent Set for Rapid Detection of Dengue Virus in Human Serum on a FieldDeployable PCR System. . Journal of clinical microbiology 56(5): e01865-17.

Tsai, Y.L., Wang, H.T., Chang, H.F., Tsai, C.F., Lin, C. K., Teng, P.H., Su, C., Jeng, C.C., Lee, P.Y., 2012. Development of TaqMan probebased insulated isothermal PCR (iiPCR) for sensitive and specific on-site pathogen detection. PLoS One 7: e45278. 